\title{
GPPS-CH-2020-102
}

\section{Numerical Investigation of Swirl-Stabilised Model Gas Turbine Combustor Using DQMoM with LES}

\author{
Yeshaswini Emmi \\ German Aerospace Centre \\ (DLR) \\ yeshaswini.emmi@dlr.de \\ Stuttgart, Germany
}

\author{
Andreas Fiolitakis \\ German Aerospace Centre \\ (DLR) \\ andreas.fiolitakis@dlr.de \\ Stuttgart, Germany
}

\author{
Manfred Aigner \\ German Aerospace Centre \\ (DLR) \\ manfred.aigner@dlr.de \\ Stuttgart, Germany
}

\begin{abstract}
A detailed numerical investigation of the swirling reactive flow in the PRECCINSTA model burner for gas turbines is carried out using the Direct Quadrature Method of Moments (DQMoM) in the context of Large Eddy Simulation (LES). DQMoM is used in this work to solve the filtered transport equations of the one-time, one-point joint thermochemical probability density function (PDF) for species and enthalpy. Transported PDF methods offer the invaluable advantage of a closed chemical source term, while DQMoM in particular provides a method for solving the PDF transport equations at reasonable computational costs: a highly valuable advantage for large, complex burners. Previous studies have examined this particular burner using LES and variety of sub-grid combustion models, chiefly with a focus on unsteady thermoacoustic modes and corresponding flow phenomena. In this work, the emphasis is placed on the flow phenomena in the stable mode, with the use of detailed chemistry due to the computational efficiency offered by DQMoM. The results are critically analysed and compared with the available experimental as well as LES model results, highlighting the particular usefulness of the DQMoM approach in the context of LES for complex burners.
\end{abstract}

\section{INTRODUCTION}

Transported probability density function (PDF) methods are an attractive approach for the modelling of turbulent reactive flows, not least since the chemical source term appears in these equations in closed form. This circumvents the need to model the closure of the chemical source. Several methods exist to solve the transported PDF equations, including stochastic field methods (Valiño, 1998) and Lagrangian Monte Carlo methods (Pope, 1985); these however tend to be relatively cost-intensive and thus intractable for most industrial applications. The Direct Quadrature Method of Moments
(DQMoM) method is an alternative approach to solving the PDF transport equations in the Eulerian framework. It retains the advantage of having a closed chemical source term while significantly reducing computational costs, allowing the model to be used for larger, more complex test cases and larger reaction mechanisms.

Several applications of the DQMoM model exist in literature (Akroyd et al., 2010; Lee and Kim, 2012; De et al., 2013; Yadav et al., 2014; Lee et al., 2015; Shen et al., 2018; Duan et al., 2018). Most, however, are restricted to the investigation of academic flames or simplified test cases. The usefulness of this model in the region of industrial combustors and complex flow regimes is yet to be fully investigated, particularly in conjunction with large eddy simulation (LES).

The present work aims to exemplify the applicability of the DQMoM model for industrial test cases containing complex geometries and flow patterns. To this end, the DQMoM model is validated in the framework of LES for the swirl-stabilised PRECCINSTA model gas turbine combustor (Meier et al., 2007). In order to quantify the accuracy of the DQMoM approach for this complex test case, comparisons are made to both experiment and further LES data, since a large number of studies using this combustor are present in the literature (Franzelli et al., 2012; Ansari et al., 2014; Wang et al., 2014; Benard et al., 2018; Fredrich et al., 2019; Verma et al., 2019).

\section{DQMOM APPROACH}

This section describes the DQMoM method for solving the transported PDF equations, and its role in the LES framework.

DQMoM is used in this work as an approach to solve the filtered one-time, one-point joint thermochemical PDF for linearly independent species mass fractions and specific enthalpy. Low Mach number and thus constant thermodynamic pressure is assumed. The PDF is filtered thus (Einstein notation is used throughout this paper): 


$$
\bar{P}\left(\psi_{\alpha} ; x_{i}, t\right)=\int_{-\infty}^{+\infty} P\left(\psi_{\alpha} ; x_{i}^{\prime}, t\right) G\left(x_{i}^{\prime}, x_{i}\right) d x_{i}^{\prime}
$$

where $\bar{P}\left(\psi_{\alpha} ; x_{i}, t\right)$ is the filtered density function (FDF) and $P\left(\psi_{\alpha} ; x_{i}, t\right)$ is the PDF, with $G\left(x_{i}^{\prime}, x_{i}\right)$ being a filter function (Pope, 2001) satisfying the condition $\int_{-\infty}^{+\infty} G\left(x_{i}\right) d x_{i}=1 . \quad x_{i}$ and $t$ denote the spatial and temporal coordinates respectively, and $\psi_{\alpha}$ is the state vector corresponding to the random vector of specific enthalpy and mass fractions, $\phi_{\alpha}$. Further, it is convenient in the context of variable density flows to use Favrefiltering:

$$
\tilde{P}\left(\psi_{\alpha} ; x_{i}, t\right)=\frac{\overline{\rho\left(\psi_{\alpha}\right) P\left(\psi_{\alpha} ; x_{l}, t\right)}}{\bar{\rho}\left(x_{i}, t\right)},
$$

with $\widetilde{(\cdot)}$ denoting a Favre-filtered quantity and $\rho$ the density. $\widetilde{P}$ is approximated in DQMoM by a finite number of Dirac pulses $\delta$ :

$$
\tilde{P}\left(\psi_{\alpha} ; x_{i}, t\right)=\sum_{n=1}^{N_{e}} p_{n}\left(x_{i}, t\right) \sum_{\alpha=1}^{N_{s}} \delta\left(\psi_{\alpha}-\bar{\phi}_{\alpha, n}\left(x_{i}, t\right)\right) .
$$

$N_{e}$ and $N_{s}$ are the number of environments and the number of scalars in the vector $\phi_{\alpha}$ respectively. $p_{n}$ is the probability of each environment $n$ and satisfies the condition $\sum_{n=1}^{N_{e}} p_{n}=1$. Now, differential diffusion is neglected and Lewis number $L e=1$; then, the transport equation for the quantity $\tilde{P}$, using a gradient diffusion model (GDM) (Pope, 1976; Pope, 1985) to close the turbulent transport terms and interaction by exchange with the mean model (IEM) (Villermaux and Devillon, 1972) to close sub-filter scale mixing, is (Colucci et al., 1998)

$$
\begin{aligned}
\frac{\partial(\bar{\rho} \tilde{P})}{\partial t}+\frac{\partial\left(\tilde{u}_{i} \tilde{\rho} \tilde{P}\right)}{\partial x_{i}}-\frac{\partial}{\partial x_{i}} & \left(\bar{\rho} D_{e f f} \frac{\partial \tilde{P}}{\partial x_{i}}\right) \\
& =-\frac{\partial}{\partial \psi_{\alpha}}\left(\left(\frac{C_{\phi}}{2 \tau_{t}}\left(\tilde{\phi}_{\alpha}-\psi_{\alpha}\right)+\frac{S_{\alpha}}{\rho}\right) \bar{\rho} \tilde{P}\right),
\end{aligned}
$$

where $\tilde{u}_{i}$ is the Favre-filtered velocity, $D_{e f f}$ is the sum of the turbulent and molecular viscosities, $C_{\phi}$ is the IEM mixing model constant and $\tau_{t}$ is the turbulent time scale. Eq. (4) is solved for using the DQMoM approach, which in summary involves forcing the definition of $\tilde{P}$ to agree with a set of known or calculable moments. Details of this method are discussed by Fox (2003). The result is a set of transport equations for the environmental probabilities $p_{n}$ and probability-weighted thermochemical scalars $\bar{s}_{\alpha, n}=$ $p_{n} \bar{\phi}_{\alpha, n}$. In this work, $N_{e}=2$ is used, and $1+2 N_{s}$ equations are obtained:

$$
\frac{\partial\left(\bar{\rho} p_{n}\right)}{\partial t}+\frac{\partial\left(\tilde{u}_{i} \bar{\rho} p_{n}\right)}{\partial x_{i}}-\frac{\partial}{\partial x_{i}}\left(\bar{\rho} D_{e f f} \frac{\partial p_{n}}{\partial x_{i}}\right)=a_{n}
$$

and

$$
\frac{\partial\left(\bar{\rho} \bar{s}_{\alpha, \mathrm{n}}\right)}{\partial t}+\frac{\partial\left(\tilde{u}_{i} \bar{\rho} \bar{s}_{\alpha, n}\right)}{\partial x_{i}}-\frac{\partial}{\partial x_{i}}\left(\bar{\rho} D_{e f f} \frac{\partial \bar{s}_{\alpha, \mathrm{n}}}{\partial x_{i}}\right)=b_{\bar{\phi}_{\alpha, n}}
$$

The right hand side (RHS) of Eq. (5) is set to zero (Fox, 2003), and that of Eq. (6) is

$$
\begin{gathered}
b_{\bar{\phi}_{\alpha, n}}=(-1)^{n} \bar{\rho} D_{e f f} \frac{p_{1} \sum_{i=1}^{3}\left(\frac{\partial \bar{\phi}_{\alpha, 1}}{\partial x_{i}}\right)^{2}+p_{2} \Sigma_{i=1}^{3}\left(\frac{\partial \bar{\phi}_{\alpha_{2}, 2}}{\partial x_{i}}\right)^{2}}{\bar{\phi}_{\alpha, 2}-\bar{\phi}_{\alpha, 1}}+ \\
\bar{\rho} p_{n} \frac{c_{\phi}}{2 \tau_{t}}\left(\tilde{\phi}_{\alpha}-\bar{\phi}_{\alpha, n}\right)+p_{n} \frac{\rho_{\alpha} s_{\alpha}}{\rho} .
\end{gathered}
$$

$S_{\alpha}$ is the chemical source term, and is not added to the enthalpy transport equations. The first term in Eq. (7) equation causes the production of scalar variance. In the case where $\bar{\phi}_{\alpha, 2}=\bar{\phi}_{\alpha, 1}$, the smoothing and bounding functions described by Akroyd et al. (2010) are used.

The Wall-Adapting Local Eddy Viscosity (WALE) (Nicoud and Ducros, 1999) model is used in this work to model the sub-grid scale stresses. The models are implemented in the DLR in-house code THETA (Reichling et al., 2013; Löwe et al., 2016), which is based on a finite-volume discretisation method. Chemistry and transport processes are handled in a coupled manner. An incompressible solver is used for the momentum equations and pressure is solved using the Projection method (Chorin, 1967).

\section{TEST CASE}

The model gas turbine combustor from the PRECCINSTA project carried out at the German Aerospace Centre (DLR) (Meier, 2007) is used in this work. A schematic of the model combustor is shown in Fig. 1. Air enters the radial swirler through the plenum at atmospheric conditions, and mixes with the fuel, $\mathrm{CH}_{4}$, which enters the swirler through 12 small injection holes. The square combustion chamber, with a cross section of $85 \times 85 \mathrm{~mm}$ and a height of $114 \mathrm{~mm}$, is constructed to allow unobstructed optical access to nearly the entire flame zone. The temperature of the fuel-air mixture entering the combustion chamber was measured to be between $T=320 \mathrm{~K}$ and $380 \mathrm{~K}$.

The complex swirling flow in this model combustor emulates that of an industrial gas turbine; it has been the subject of several numerical investigations (see references in Introduction) and thus a large dataset is available for the purpose of model validation. The experiment details are described in the following subsection, after which the numerical set-up for this work is laid out.

\section{Experiment}

A number of measurements were performed in this investigation: flow velocities were determined through Laser Doppler Velocimetry (LDV), joint PDFs of major species concentrations, temperature and mixture fraction by laser Raman scattering, and flame shape and heat release zones through $\mathrm{OH}$ chemiluminescence. Two types of flame were studied: a stable one operating at $30 \mathrm{~kW}$ and an equivalence ratio of $\phi=0.83$, and a pulsating, unstable one operating at $25 \mathrm{~kW}$ and an equivalence ratio of $\phi=0.70$. 


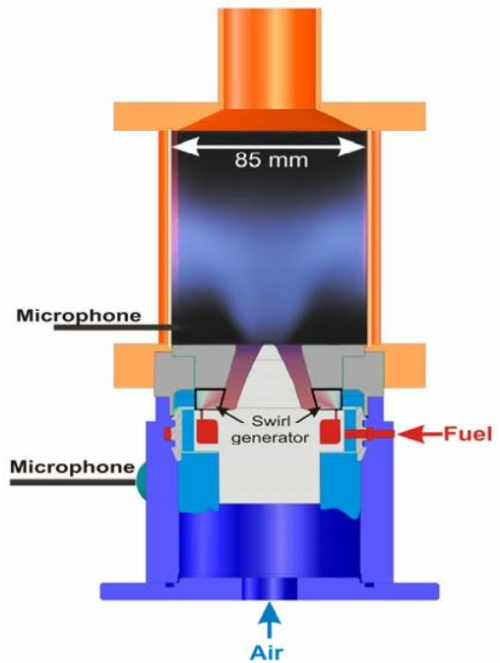

Figure 1. Schematic of the experimental set-up (Meier, 2007).

\section{Numerical Set-Up}

In this work, the stable flame $(\phi=0.83$; Reynold's number $R e \approx 31,000$ ) from the experiments is investigated. The computational domain used in this work is identical to that presented in Lourier et al.'s (2017) work. An unstructured, tetrahedral mesh with wall prism layers making up approximately 8 million nodes is used. Five prism layers coat the bluff body leading into the combustion chamber, and three the combustion chamber walls; here, the first prism layer sits at $y^{+}<1$. The grid spacing $\Delta x$ varies widely throughout the domain. In the fuel injection region, $\Delta x \approx 0.04 \mathrm{~mm}$; in the flame region, $\Delta x \approx 0.75 \mathrm{~mm}$; and in the plenum region, $\Delta x \approx 3 \mathrm{~mm}$. A constant time step of $\Delta t=2 \times 10^{-7} \mathrm{~s}$ is applied. This corresponds to the CFL number being less than 0.03 in the flame regions and less than 0.4 in the fuel injection and swirler regions. Further, the Kolmogorov length scale approximated by Benard et. al (2018) at $\eta \approx 29 \mu \mathrm{m}$ corresponds to a grid spacing that is about 26 times the value of $\eta$ in the flame region. A total of approximately 60,000 core-hours were spent on this calculation.

Finite rate chemistry is employed; detailed chemical kinetics is used to model the methane oxidisation process, with 19 reacting and 2 non-reacting species, and 84 reactions (Kazaov and Frenklach, 1994). The WALE model constant $C_{W}=0.4$ is used.

Table 1. Boundary conditions.

\begin{tabular}{llll}
\hline & & Inlet Fuel & Inlet Air \\
\hline Mass flow rate & {$[\mathrm{g} / \mathrm{s}]$} & 5.983 & 12.237 \\
\hline Species & {$[-]$} & $\mathrm{CH}_{4}$ & $\mathrm{O}_{2}, \mathrm{~N}_{2}$ \\
\hline Mass Fractions & {$[-]$} & 1.0 & $0.23,0.77$ \\
\hline Temperature & {$[\mathrm{K}]$} & 320 & 320 \\
\hline $\boldsymbol{p}_{\mathbf{1}}$ & {$[-]$} & 0.2 & 0.2 \\
\hline
\end{tabular}

\section{DQMoM Boundary Treatment}

The boundary conditions are described in Table 1. The inlet temperature is set to the lower bound of the measured data: although this lower preheating value may have a moderate effect on the results, it is chosen in order to keep consistent with LES studies in literature. Additionally, since the inclusion of wall heat losses have a significant impact on the flame dynamics (Benard et al, 2018), the walls of the combustion chamber and the conical bluff body leading into the combustion chamber are modelled as isothermal. The combustion chamber walls are set to $1800 \mathrm{~K}$, while the walls of the conical bluff body upstream of the combustion chamber are at $1200 \mathrm{~K}$.

The environmental probabilities at the inflow boundaries are set to $p_{1}=0.2$ and $p_{2}=0.8$, with the species mass fractions of each environment, $\bar{Y}_{\alpha, i n, n}$, perturbed about the mean, $\widetilde{Y}_{\alpha, \text { in }}$ :

$$
\bar{Y}_{\alpha, \mathrm{in, \textrm {n }}}=\tilde{Y}_{\alpha, i n}+(-1)^{n} \frac{1 \times 10^{-4}}{p_{i n, n}} .
$$

The subscript $(\cdot)_{\text {in }}$ denotes an inflow variable. This formulation requires that at each inlet, the two environments are identical to the mean physical thermochemical state at that inlet; but, since identical environmental values produce a singularity in the variance production term (see Eq. (7)), the environmental values are perturbed about this mean physical state. Eq. (8) ensures that the mean is preserved while allowing sensible values for the variance production term. Further details of this treatment, as well as the handling of isothermal walls within the DQMoM model, can be found in the work by Emmi et al. (2019).

\section{RESULTS AND DISCUSSION}

The following section presents the results obtained from the DQMoM simulation and compares them to values from experiment. A general idea of the flow can be ascertained from Fig. 2. A large inner recirculation zone and smaller recirculation regions on either side of the inflow jets, bounded by the walls, is seen. Fig. 3 shows the instantaneous Favre-filtered $\mathrm{OH}$ mass fraction distribution. A V-shaped flame that penetrates a little under $40 \mathrm{~mm}$ into the combustion chamber is observed; it extends marginally upstream of the inlet on the head of the conical bluff body.

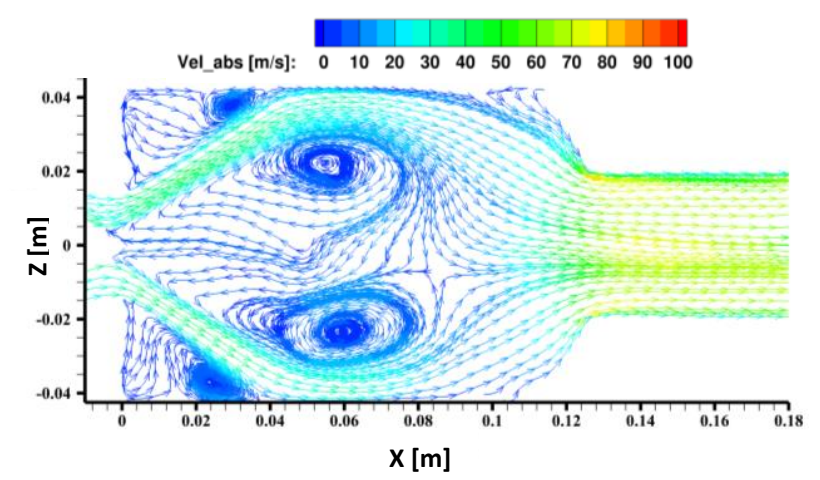

Figure 2. Time-averaged, Favre-filtered velocity streamlines, coloured by velocity magnitude. Slice from combustion chamber midplane. 


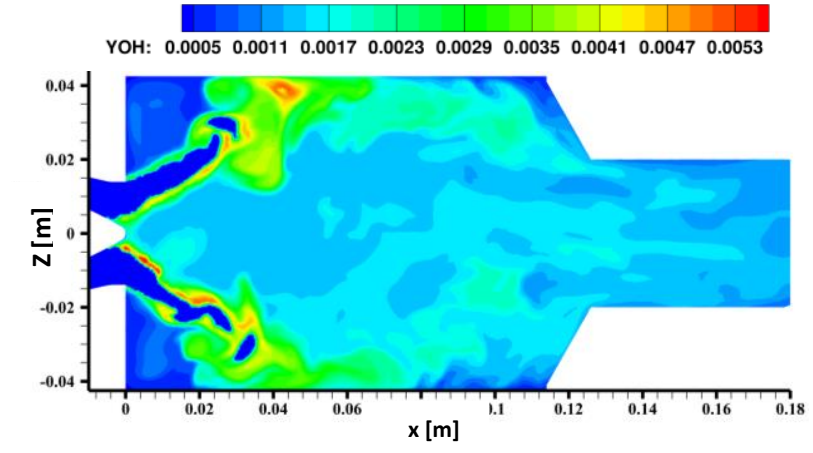

Figure 3. Instantaneous Favre-filtered $\mathrm{OH}$ mass fraction. Slice from combustion chamber midplane.
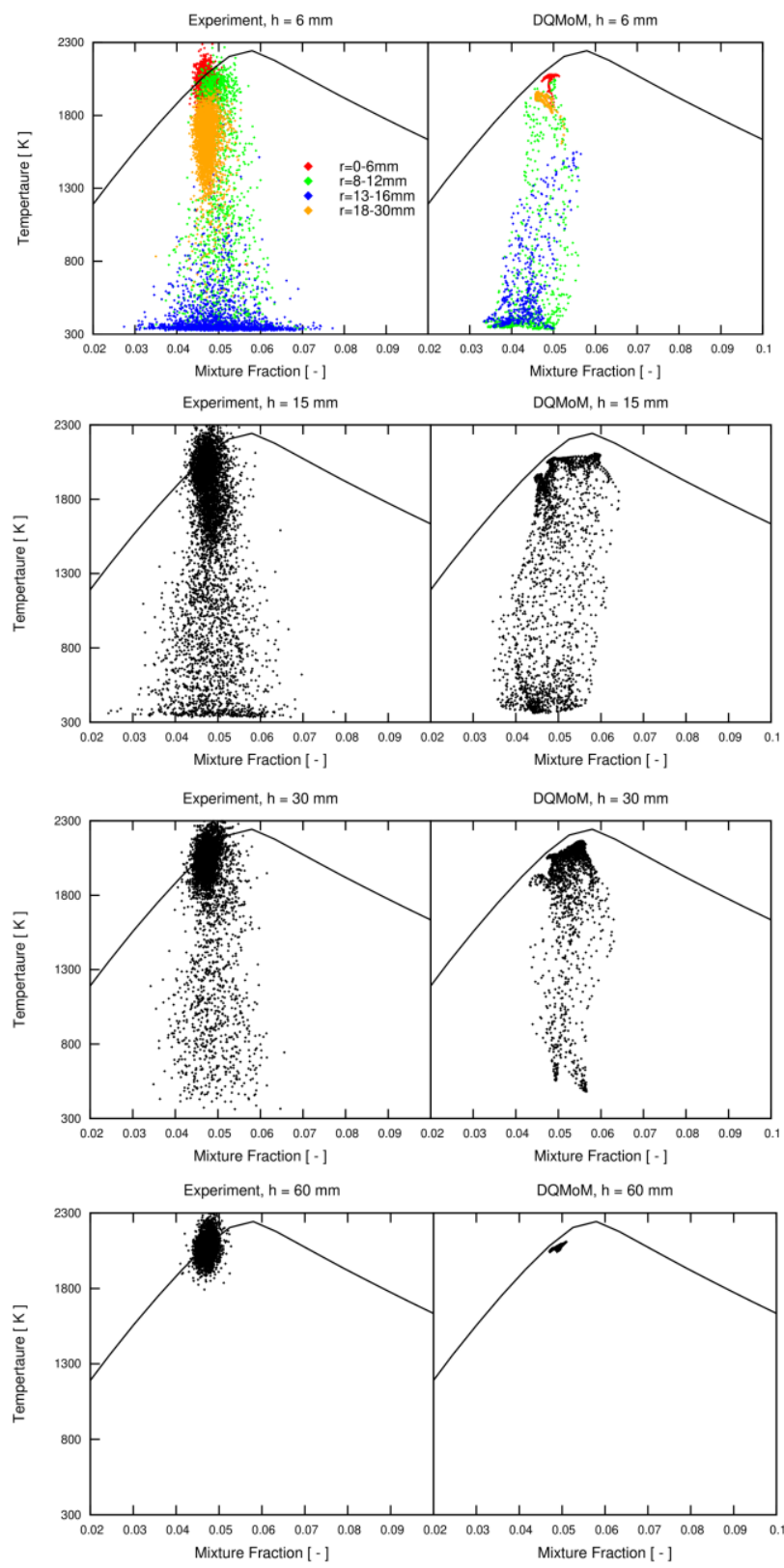

Figure 4. Instantaneous mixture fraction vs temperature at 4 positions downstream of the combustion chamber inlet, for various radial positions. Experiment on the left and simulation on the right.
Fig. 4 plots temperature against mixture fraction for various positions downstream of the combustion chamber inlet, with experiment on the left and DQMoM on the right. The black line indicates adiabatic flame temperature. In general, the scatter plots from the simulation emulate the experimental data. Notably, however, for $h=6 \mathrm{~mm}$ (Fig. 4 top), the computed scatter in mixture fraction appears to be narrower for low temperatures. At this location, the computed mixture fraction is shifted toward an overall leaner mixture, particularly for $r=13-16 \mathrm{~mm}$. This encompasses approximately the outer shear layer and suggests that the level of premixing is overpredicted by the simulation. This is further evidenced by the narrow range of mixture fractions also in the jet region $(r=8-12 \mathrm{~mm})$ Approaching the wall at $h=6 \mathrm{~mm}$, for $r>18 \mathrm{~mm}$, the temperature range as well as the mixture fraction range is smaller in the numerical data, which indicates a greater presence of hot burnt gases that have not mixed with fresh gases. Interestingly, at $h=15 \mathrm{~mm}$, the mixture fraction range at higher temperatures is larger in the simulation than in the experiment. The presence of peak temperatures in richer mixture fractions at this location may indicate once again that there are regions of incomplete mixing between fresh and burnt gas (compared to experiment), where ignition still occurs. This relatively rich combustion has the tendency to be incomplete (see overprediction in CO mass fraction in Fig. 8). As a consequence, the equilibrium temperature is also not quite
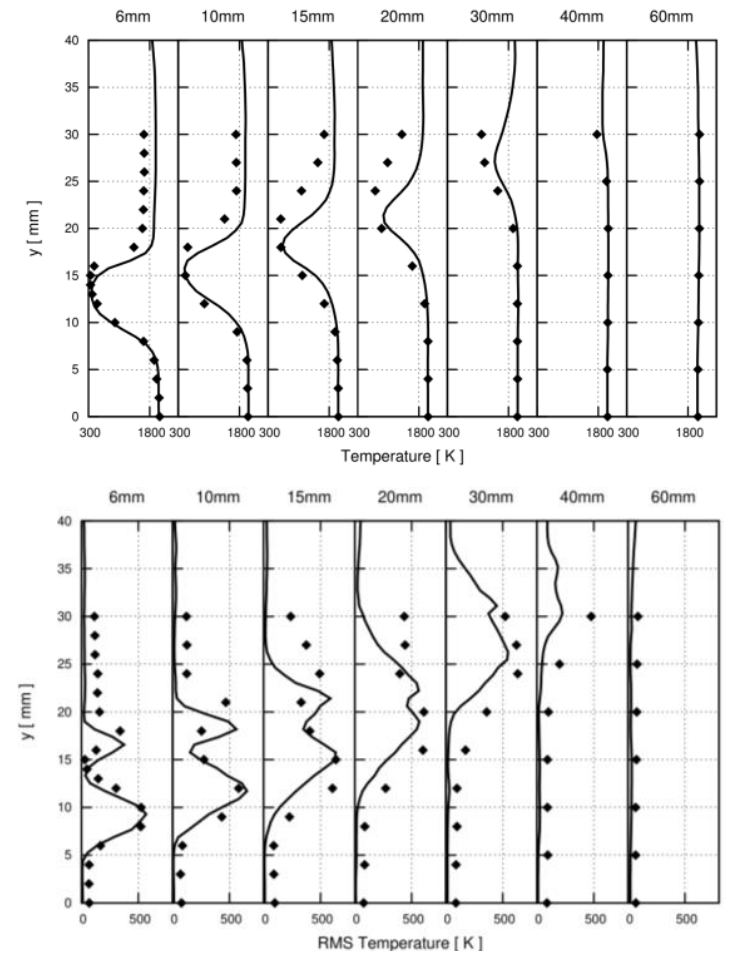

Figure 5. Time-averaged, Favre-filtered temperature (top) and RMS temperature (bottom) profiles at several positions downstream of the combustion chamber. Profiles from centreline to wall. Diamonds: experiment, lines: DQMoM. 
reached. The trend continues down to $h=30 \mathrm{~mm}$, but by $h=60 \mathrm{~mm}$, the peak temperatures align again with experiment in the mixture fraction space. The inclusion of wall heat losses also contribute to the equilibrium temperature not being reached. The equilibrium temperature is reached and maintained in the experiment in the inner flame zone but peak temperatures drop in the outer flame regions since fresh gas mixing is present. Data points for lower temperatures disappear for consecutive plots in Fig. 4 slightly sooner in the DQMoM data, indicating fewer unreacted states in the simulation than the experiment. A fully burnt state is reached by $h=60$ $\mathrm{mm}$, while the experiment still shows a marginally higher range of temperatures and mixture fractions at this location.

Fig. 5 (top) shows Favre-filtered, time-averaged temperature profiles in the combustion chamber at seven different heights starting at $6 \mathrm{~mm}$ from the base of the combustion chamber up to $60 \mathrm{~mm}$ downstream of it. At each of these axial locations, values are extracted from the centre of the combustion chamber up to $40 \mathrm{~mm}$ in the radial direction. The time-averaging has been performed over six residence times through the combustion chamber. The values for temperature agree fairly well; however, some deviation is evident at upstream locations and approaching the wall. Firstly, the narrower "well" in the simulation profiles indicates a higher degree of mixing of the hot exhaust gases with the fresh unburnt gases than in the experiment; there are thus higher temperatures
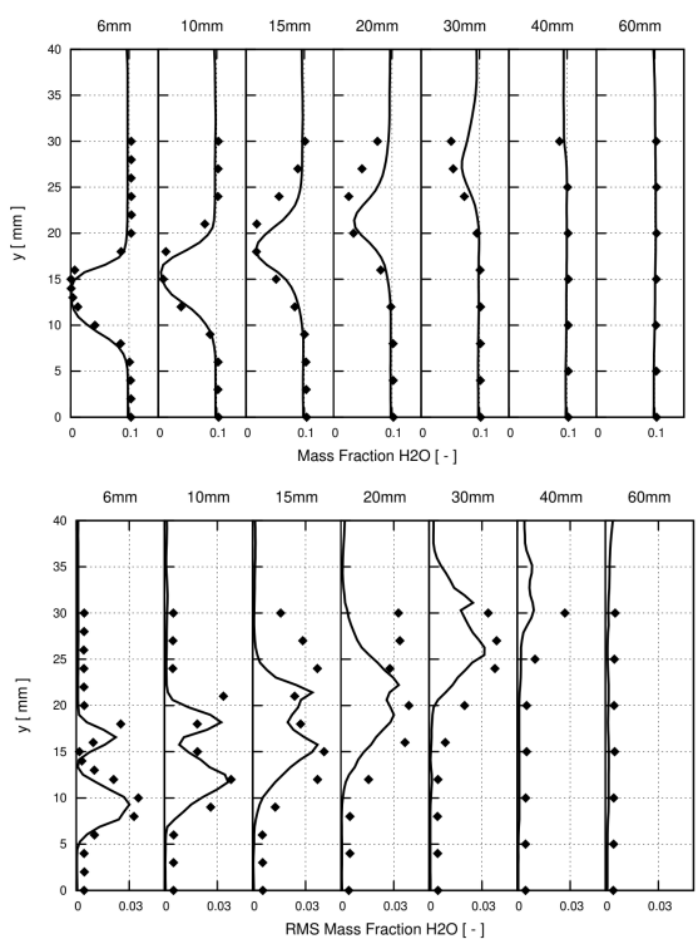

Figure 6. Time-averaged, Favre-filtered $\mathrm{H}_{2} \mathrm{O}$ mass fraction (top) and RMS (bottom) profiles at several positions downstream of the combustion chamber. Profiles from centreline to wall. Diamonds: experiment, lines: DQMoM.
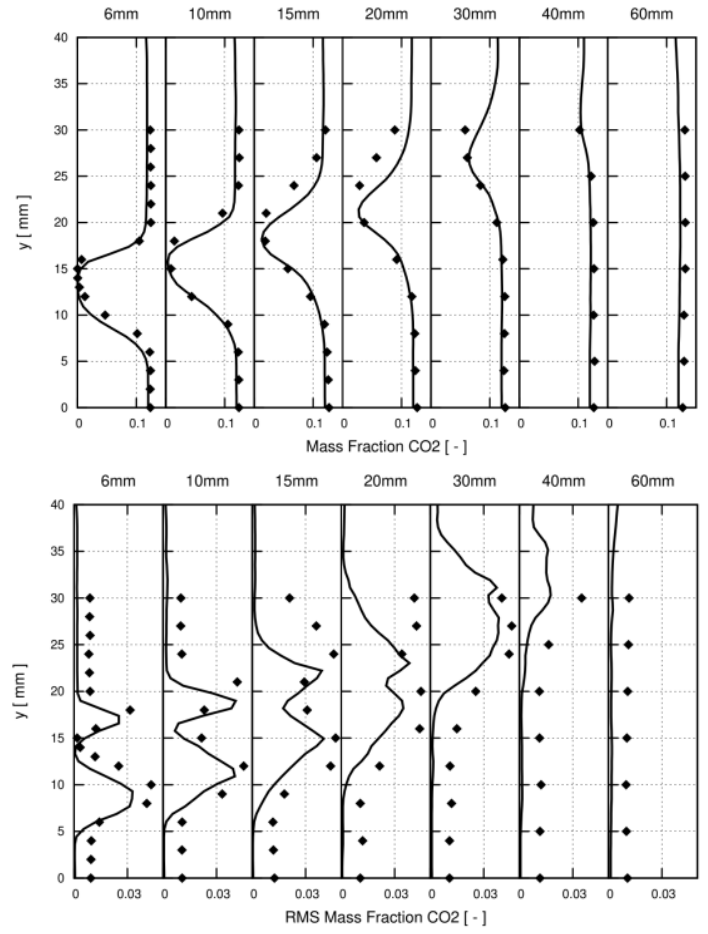

Figure 7. Time-averaged, Favre-filtered $\mathrm{CO}_{2}$ mass fraction (top) and RMS (bottom) profiles at several positions downstream of the combustion chamber. Profiles from centreline to wall. Diamonds: experiment, lines: DQMoM.
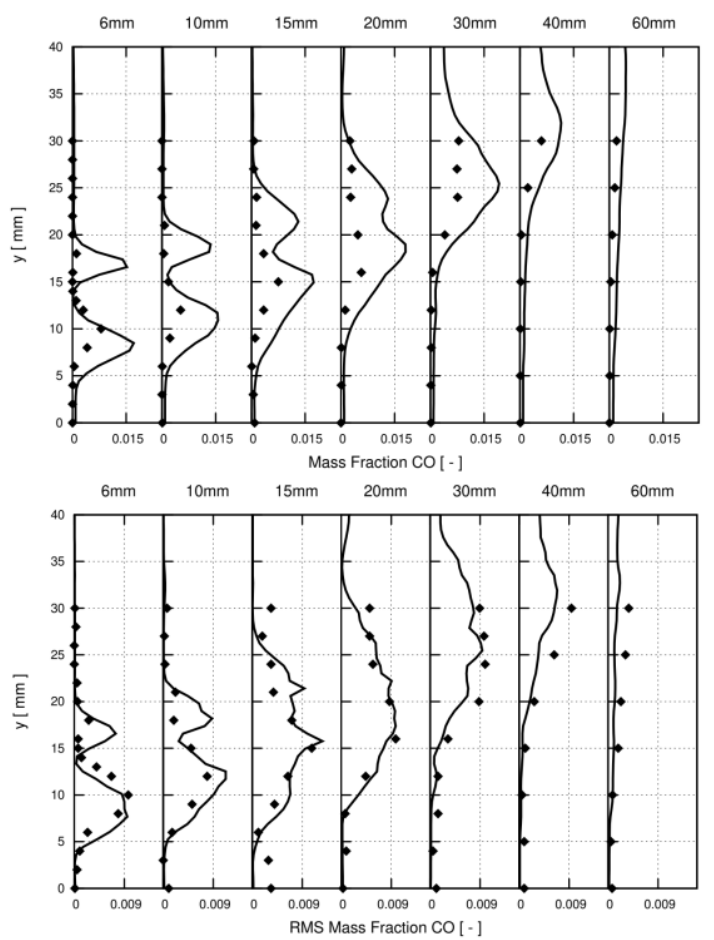

Figure 8. Time-averaged, Favre-filtered CO mass fraction (top) and RMS (bottom) profiles at several positions downstream of the combustion chamber. Profiles from centreline to wall. Diamonds: experiment, lines: DQMoM. 
predicted by DQMoM past the well. This follows from the trends already seen in Fig. 4. Inaccurate reproduction of the wall temperature distribution could also be a potential factor in this $100-200 \mathrm{~K}$ difference between simulation and experiment. The root mean square (RMS) of temperature (both resolved and sub-grid from the simulation) is compared to experiment in Fig. 5 (bottom). Both shape and magnitude of the RMS distribution are reproduced very well, although the simulation predicts a drop in RMS in the outer region of the flame bounded by the wall rather steeply. This again follows from the discussion for Fig. 4, where the hot burnt gases in this region dominate with very low presence of fresh gas.

Figs. 6, 7 and 8 (top) plot the Favre-filtered, timeaveraged mass fractions of $\mathrm{H}_{2} \mathrm{O}, \mathrm{CO}_{2}$ and $\mathrm{CO}$ respectively, while Figs. 6, 7 and 8 (bottom) show the corresponding RMS values (again a summation of filtered and sub-grid values for DQMoM). The general trends of the mass fraction data of $\mathrm{CO}_{2}$ and $\mathrm{H}_{2} \mathrm{O}$ are consistent between DQMoM and experiment. At axial locations of $15 \mathrm{~mm}$ and $20 \mathrm{~mm}$, some deviation is observed, particularly between $20 \mathrm{~mm}$ and $30 \mathrm{~mm}$ in the radial direction. $\mathrm{CO}_{2}$ and $\mathrm{H}_{2} \mathrm{O}$ are slightly overpredicted here. This follows from the overprediction of temperature in these regions and is likely related to the inadequate level of mixing at these locations, since both species match the experimental data very well outside of this range. The $\mathrm{CO}$ profiles from simulation follow the trends from experiment fairly well, though a thorough comparison is difficult due to a high error margin $(\sim 50 \%)$ in the experiment for this quantity. The double peak appearing in Fig. 8 at upstream locations is consistent with LES in literature (Benard, 2018; Ansari, 2014; Wang, 2014). The RMS values for the mass fractions are consistently slightly underpredicted, particularly at locations downstream of $20 \mathrm{~mm}$ and, in the case of $\mathrm{H}_{2} \mathrm{O}$ and $\mathrm{CO}_{2}$, in the outer flame region. This follows from the previous discussion pertaining to low temperature RMS and inadequate mixing as well.

\section{CONCLUSIONS}

The DQMoM model has been validated in this work using LES for a model gas turbine combustor consisting of complex geometries and flow patterns. The results suggest that the DQMoM model performs well when compared to the experimental data. The most prominent deviations are observed in the region between the flame and the wall. Here, the mixing between the fresh unburnt gases and the hot burnt gases is not captured accurately by the simulation. Additionally, the imperfect premixing present in the experiment is not particularly well reproduced in the simulation. This is also seen by other LES data (Fredrich et al., 2019). The inadequate degree of scalar mixing could be a consequence of the micro mixing model and the corresponding model constants chosen for this work. Further investigation of the influence of this parameter, as well as the chosen mixing model itself, would be instructive in this regard.
Overall, this work suggests that DQMoM could be an attractive model for the simulation of industrial combustors. The computational costs involved are several-fold lower than those associated with the stochastic approaches to TPDF simulations, for example Lagrangian Monte Carlo or stochastic field methods. The results obtained by DQMoM are comparable to those reported by Ansari et al. (2014) (though still somewhat less accurate), who employed a Lagrangian Monte-Carlo approach to solve the transported filtered density function equations. The discrepancies between experiment and simulations are also lower than those reported for example by Wang et al. (2014), who employ a presumed-PDF approach with LES; particularly for the values of RMS. In light of these observations, the DQMoM model can be recommended for use in the further numerical investigation of industrial combustors.

\section{REFERENCES}

\section{Articles}

Ansari, N., Strakey, P.A., Goldin, G.M. and Givi, P., 2015. Filtered density function simulation of a realistic swirled combustor. Proceedings of the Combustion Institute, 35(2), pp.1433-1442.

Akroyd, J., Smith, A.J., McGlashan, L.R. and Kraft, M., 2010. Numerical investigation of DQMoM-IEM as a turbulent reaction closure. Chemical Engineering Science, 65(6), pp.1915-1924.

Bénard, P., Lartigue, G., Moureau, V. and Mercier, R., 2019. Large-Eddy Simulation of the lean-premixed PRECCINSTA burner with wall heat loss. Proceedings of the Combustion Institute, 37(4), pp.5233-5243.

Chorin, A.J., 1967. The numerical solution of the NavierStokes equations for an incompressible fluid. Bulletin of the American Mathematical Society, 73(6), pp.928-931.

Colucci, P.J., Jaberi, F.A., Givi, P. and Pope, S.B., 1998. Filtered density function for large eddy simulation of turbulent reacting flows. Physics of Fluids, 10(2), pp.499-515.

De, A., Dongre, A. and Yadav, R., 2013. Numerical investigation of delft-jet-in-hot-coflow (DJHC) burner using probability density function (PDF) transport modeling. In ASME Turbo Expo 2013: Turbine Technical Conference and Exposition. American Society of Mechanical Engineers Digital Collection.

Duan, X., Feng, X., Yang, C. and Mao, Z., 2018. CFD modeling of turbulent reacting flow in a semi-batch stirred-tank reactor. Chinese Journal of Chemical Engineering, 26(4), pp.675-683. 
Emmi, Y., Fiolitakis, A., Aigner, M., Genin, F. and Syed, K., 2019. A New Model Approach for Convective Wall Heat Losses in DQMOM-IEM Simulations for Turbulent Reactive Flows. Journal of Engineering for Gas Turbines and Power, 141(5).

Franzelli, B., Riber, E., Gicquel, L.Y. and Poinsot, T., 2012. Large eddy simulation of combustion instabilities in a lean partially premixed swirled flame. Combustion and Flame, 159(2), pp.621-637.

Fredrich, D., Jones, W.P. and Marquis, A.J., 2019. The stochastic fields method applied to a partially premixed swirl flame with wall heat transfer. Combustion and Flame, 205, pp.446-456.

Lee, J. and Kim, Y., 2012. DQMOM based PDF transport modeling for turbulent lifted nitrogen-diluted hydrogen jet flame with autoignition. International Journal of Hydrogen Energy, 37(23), pp.18498-18508.

Lee, J., Jeon, S. and Kim, Y., 2015. Multi-environment probability density function approach for turbulent $\mathrm{CH} 4 / \mathrm{H} 2$ flames under the MILD combustion condition. Combustion and Flame, 162(4), pp.1464-1476.

Lourier, J.M., Stöhr, M., Noll, B., Werner, S. and Fiolitakis, A., 2017. Scale Adaptive Simulation of a thermoacoustic instability in a partially premixed lean swirl combustor. Combustion and Flame, 183, pp.343357.

Löwe, J., Probst, A., Knopp, T. and Kessler, R., 2016. Low-dissipation low-dispersion second-order scheme for unstructured finite volume flow solvers. AIAA Journal, pp.2961-2971.

Meier, W., Weigand, P., Duan, X.R. and GiezendannerThoben, R., 2007. Detailed characterization of the dynamics of thermoacoustic pulsations in a lean premixed swirl flame. Combustion and Flame, 150(1-2), pp.2-26.

Nicoud F, Ducros F., 1999. Subgrid-scale stress modelling based on the square of the velocity gradient tensor. Flow, Turbulence and Combustion. 62(3):183200.

Pope, S.B., 1976. The probability approach to the modelling of turbulent reacting flows. Combustion and Flame, 27, pp.299-312.

Pope, S.B., 1985. PDF methods for turbulent reactive flows. Progress in Energy and Combustion Science, 11(2), pp.119-192.

Reichling, G., Noll, B. and Aigner, M., 2013. Development of a projection-based method for the numerical calculation of compressible reactive flows. In 51st AIAA Aerospace Sciences Meeting including the New Horizons Forum and Aerospace Exposition (p. 1003).

Shen, H., Wu, Y., Xu, K., Smith, P.J. and Zhang, H., 2018. Eulerian LES simulation of coal jet flame with a simplified DQMOM model. Fuel, 216, pp.475-483.

Valino, L., 1998. A field Monte Carlo formulation for calculating the probability density function of a single scalar in a turbulent flow. Flow, Turbulence and Combustion, 60(2), pp.157-172.

Verma, I., Yadav, R., Sharkey, P., Li, S. and Meeks, E., 2019, June. Modeling of Turbulent Swirl Stabilized Flame With Flamelet Generated Manifold and Hybrid LES-RANS Turbulence Model. In Turbo Expo: Power for Land, Sea, and Air (Vol. 58622, p. V04BT04A011). American Society of Mechanical Engineers.

Villermaux, J. and Devillon, J.C., 1972, May. Représentation de la coalescence et de la redispersion des domaines de ségrégation dans un fluide par un modèle d'interaction phénoménologique. In Proceedings of the 2nd International Symposium on Chemical Reaction Engineering (Vol. 26, pp. 1-13). Elsevier New York.

Wang, P., Platova, N.A., Fröhlich, J. and Maas, U., 2014. Large eddy simulation of the PRECCINSTA burner. International Journal of Heat and Mass Transfer, 70, pp.486-495.

Yadav, R., Kushari, A., Eswaran, V. and Verma, A.K., 2014. A detailed validation study of multi-environment eulerian probability density function transport method for modeling turbulent nonpremixed combustion. Journal of Engineering for Gas Turbines and Power, 136(8).

\section{Books}

Fox, R.O., 2003. Computational models for turbulent reacting flows. Cambridge University Press.

Pope, S.B., 2001. Turbulent flows. Cambridge University Press.

\section{Websites}

Kazakov, A. and Frenklach, M., 1994. Reduced reaction sets based on GRI-Mech 1.2. University of California at Berkeley, Berkeley, CA, http://www. me. berkeley. edu/drm. 